\title{
Helping Teachers Visualize Students' Performance
}

\author{
Ranilson Paiva, Ig Ibert Bittencourt, Wansel Lemos \\ ${ }^{1}$ Computing Institute - Federal University of Alagoas (UFAL) \\ Maceió, AL - Brazil \\ \{ranilsonpaiva, ig.ibert\}@ic.ufal.br, wansellemos@gmail.com
}

\begin{abstract}
The growth in the number of on-line courses evidences a new paradigm where education is available anywhere and anytime (and, hopefully, to any person). In this new paradigm Courses occur in On-line learning environments, which rely on information and communication technology to promote learning and teaching. However, researchers report that, on average, $85 \%$ of students drop out these courses, and they blame the lack of teacher's support. In this regard, it is necessary to help teachers analyze the data these environments generate, extracting relevant information to guide their decisions. Learning Analytics, Educational Data Mining and Data Visualization can be used to deal with these data, but training teachers on these techniques would demand them time and effort, and the effectiveness is unknown. We propose, instead, the use of DataViz to help teachers aggregate and "see" their students' according to their performance level. We asked teachers to interact with some visualizations. We then checked if they understood the information presented, and asked about their perceptions regarding the: utility, ease of use, attitude towards use, intention to use, aesthetics, the color scheme used and the vocabulary used. The results indicate teachers understood and had positive perceptions regarding the visualizations used.
\end{abstract}

Keywords: Data Visualization, Educational Data Mining, Learning Analytics and Pedagogical Decision-Making.

\section{Introduction}

The constant growth in the number on-line courses [Allen et al. 2016] evidences a new educational paradigm. In it, classes happen in on-line learning environments, where much of the classroom activities happens at flexible times, available to those with an Internet access (AA Learning ${ }^{1}$ ). This paradigm, however, demands the use of digital information and communication technologies (DICT).

These facts bring some challenges, such as overcoming the considerable amount of learners who dropout from on-line courses $(85 \%$, on average, according to Onah et al. 2014, Liyanagunawardena et al. 2014). These learners blame the "Lack of Instructor Support" as a reason for quitting the course Onah et al. 2014, Liyanagunawardena et al. 2014, thus indicating the need to help teachers dealing with this situation. Nevertheless, to provide such support, teachers would have to be able to analyze the data generated by the learning environ-

\footnotetext{
${ }^{1}$ AA Learning: Anywhere and anytime learning (for any person) Bittencourt et al. 2009]
} 
VIII Congresso Brasileiro de Informática na Educação (CBIE 2019)

Anais do XXX Simpósio Brasileiro de Informática na Educação (SBIE 2019)

ment in order to extract useful information to guide pedagogical decision-making Bienkowski et al. 2012, Schildkamp et al. 2012.

Learning Analytics (LA), Educational Data Mining (EDM) and Data Visualization (DataViz) are techniques used for these analyses. LA collects learners' data to improve learning [Baker et al. 2012]. EDM processes these data to find patterns or computing indicators [Baker et al. 2012. DataViz converts data/information into a representative image for human viewing, helping quick access and understanding ${ }^{2}$.

However, training teachers on these techniques demand investments in time and effort Bienkowski et al. 2012, Schildkamp et al. 2012, and the effectiveness is not garanteed. Thus, there is a latent need to help teachers extracting relevant information from the educational data in order to guide pedagogical decisionmaking. Ideally, this information should be easy to understand and visually appealing to teachers, allowing them to (quickly) perceive what is happening with their learners and also guide them on what to do (pedagogical decision-making) [Bienkowski et al. 2012, Schildkamp et al. 2012, Romero and Ventura 2016].

Considering these information, we identify the following business problem: how can we support teachers' understanding of what is happening with their students? As technical problem: how can we use data visualization to help teachers understand the output of educational data processing? Based on these problems, we defined the following research goal: create visualizations to help teachers understand the output from educational data processing and analysis. To reach this goal, we created 4 data visualizations to simplify the analysis of: (1) a bar graph; (2) the output of the SimpleLogistic algorithm; (3) the output of the JRIP ; and (4) the output of the J48 algorithms ${ }^{3}$,

We evaluated the proposal checking if teachers understood the visualization, and asked their perceptions about the visualizations, via a questionnaire, regarding the following metrics: utility, ease of use, attitude towards use, intention to use, aesthetics, the color scheme used and the vocabulary used. 116 teachers participated in the experiment. They were able to understand the purpose of the visualizations, with Visualization 4 having the higher "understandability" score. Regarding the participants' perception on the metrics mentioned the median of their responses was at least 4 (in a Likert scale from 0 to 6), which represents the visualizations were perceived as useful, easy to use, interesting, the teachers would use them if they were available, beautiful and the color scheme was appropriate. The results also show that the vocabulary used needs to be improved.

\section{Proposal}

We propose the use of data visualization to help teachers understand the output from the use of data mining and learning analytics on students' interactions data (problems solved correctly, incorrectly and in total, accesses to learning environment, videos watched, points, trophies and level).

\footnotetext{
${ }^{2}$ As defined in: https://businessintelligence.com/dictionary/data-visualization/

${ }^{3}$ These algorithms are implemented in WEKA version 3.8.
} 


\subsection{Visual Support}

We created 4 visualizations named: Segmented Bar Graph (Figure 1); Ordered Weights (Figure 2); Combined Interactions (Figure 3); Interaction Routes (Figure 4). Each visualization addressed a particular technique from Educational Data Mining or Learning Analytics, and were created based on interactional data from 196 students (9 months of interactions in an online learning environment). Each visualization has the following general objectives: (1) allow teachers to understand what is happening with their learners; (2) group learners according to their results; (3) support pedagogical decisions based on (visual) evidences.

On-line learning environments support large numbers of learners interacting simultaneously. Thus, it may be impossible for teachers to deal individually with each student, so we applied a technique called "RAG4 Colors" Alexander et al. 2014]. The objective was to aggregate students into classes according to their interactions: (1) Inadequate class (Red): these learners need immediate/urgent attention and well-planned pedagogical interventions Alexander et al. 2014. If nothing is done, there is a high probability these learners will fail or feel abandoned/discouraged and drop out of the course Onah et al. 2014, Liyanagunawardena et al. 2014; (2) Insufficient class (Yellow/Amber): these learners need attention and monitoring [Alexander et al. 2014. Their situation is better than those in the inadequate class, but they need to progress to the Adequate class; (3) Adequate Class (Green): these learners need incentives and/or challenges to keep them motivated and progressing well. Teachers can recommend interactions from students in this class.

Visualization 1 - Segmented Bar Graph In the segmented bar graph displays the students' interactions are counted and compared to the mean of all interactions of the same type. Learners with scores below -1 standard deviation, were placed in the inadequate class (red); those with scores between -1 and +1 standard deviation, were placed in the insufficient class (amber/yellow); and those with scores above +1 standard deviation, were placed in the adequate class (green). The objective was to isolate classes of results and facilitate comparison (Figure 1).

Visualization 2 - Ordered Weights For the creation of this visualization, we ran the SimpleLogisti $\complement^{5}$ algorithm on the educational data. The output is informative, but it is not "teacher-friendly". We used the output to create the "Ordered Weights" visualization, which shows the impact of the learners' interactions in their overall performance (Figure 2).

We considered the coefficients of each interaction (variable) in the output. Interaction with negative means repel learners from a class. For instance, for the inadequate class, negative coefficients are desirable and positive variables are undesirable. Thus, we calculated how much each interaction positively impacts on the learners' performance. We ordered these variables (greatest to least impact) and

\footnotetext{
${ }^{4} \mathrm{RAG}$ stands for: red, amber, green.

${ }^{5} \mathrm{~A}$ classifier that constructs linear regression models [Sumner et al. 2005].
} 
VIII Congresso Brasileiro de Informática na Educação (CBIE 2019)

Anais do XXX Simpósio Brasileiro de Informática na Educação (SBIE 2019)

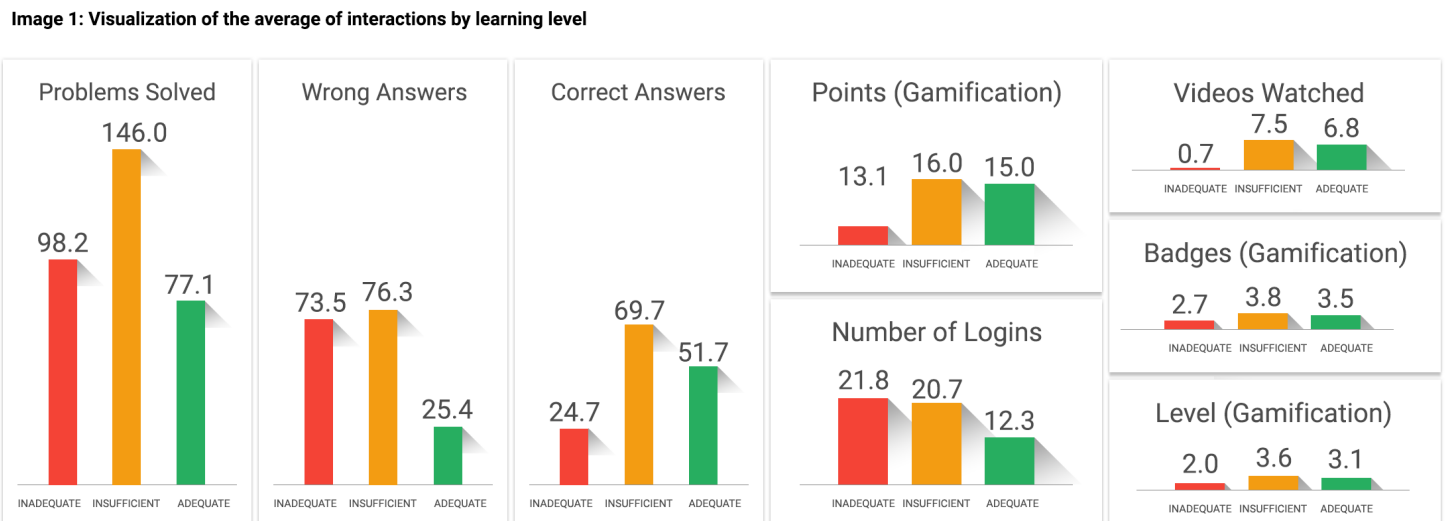

Figure 1. Viz 1 - Segmented Bar Graph, based on a traditional bar graph.

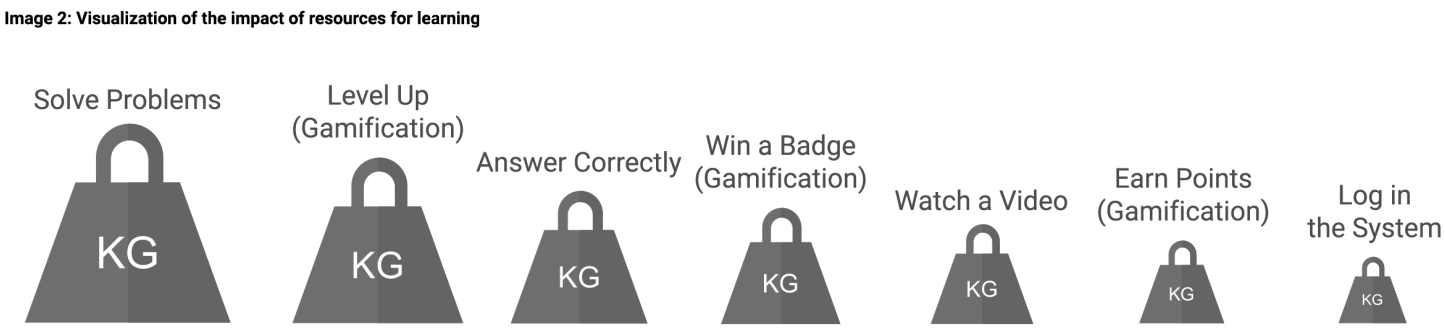

Figure 2. Viz 2 - Ordered Weights, based on WEKA's Simple Logistic output.

transformed into weight designs of different sizes, whose areas correspond to the impact.

Visualization 3 - Combined Interactions For the creation of this visualization, we ran the JRin 6 . The output shows some combinations of interactions leading to a particular class of results. The text output is not easy to understand. We used the output to create the "combined interactions" visualization, which shows the association of interactions that lead to the inadequate class and the interactions that lead to the adequate class (Figure 3).

Each interaction type was represented by an icon and green arrows, and red arrows, indicate that these types of interaction promote or damage students' performance, respectively. We calculated a scored for each type of interaction. For each occurrence in the association rules outputted, a point was summed to the score. If a type of interaction did not occur in a rule, a point was subtracted from the score.

Visualization 4 - Course of Interactions For the creation of this visualization, we ran the $J 48$ algorithm on the educational data. WEKA allows the output to be displayed as a Decision Tree. However, interpreting it is not be an easy task for teachers. We used the results to create the "course of interactions" visualization,

\footnotetext{
${ }^{6}$ It infers association rules [Cohen 1995] based on frequent and relevant patterns occurring within the data.
} 
VIII Congresso Brasileiro de Informática na Educação (CBIE 2019)

Anais do XXX Simpósio Brasileiro de Informática na Educação (SBIE 2019)

Image 3: Visualization of the influence of interactions for learning.

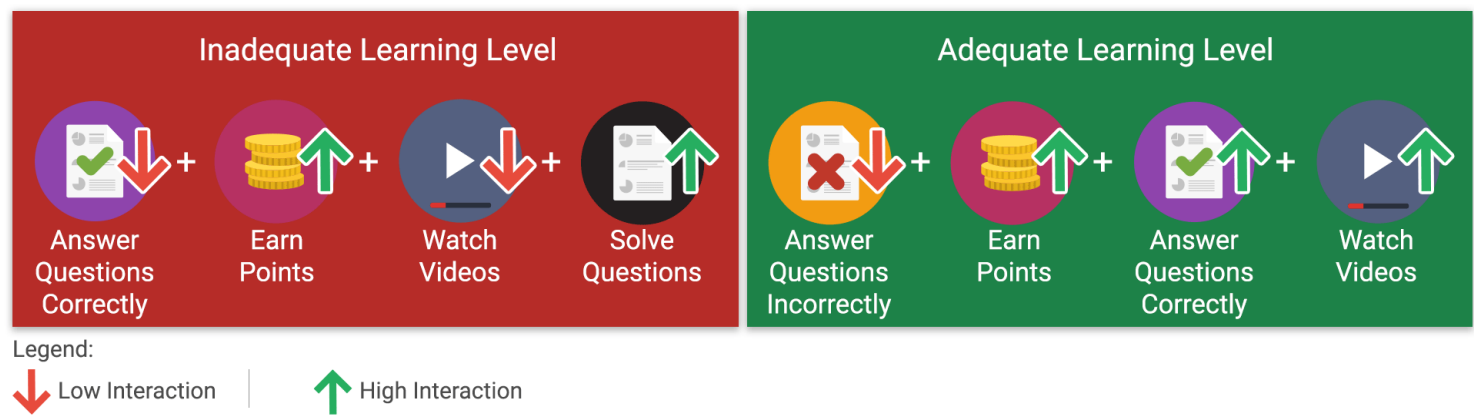

Figure 3. Viz 3 - Combined Interactions, based on WEKA's JRip output.

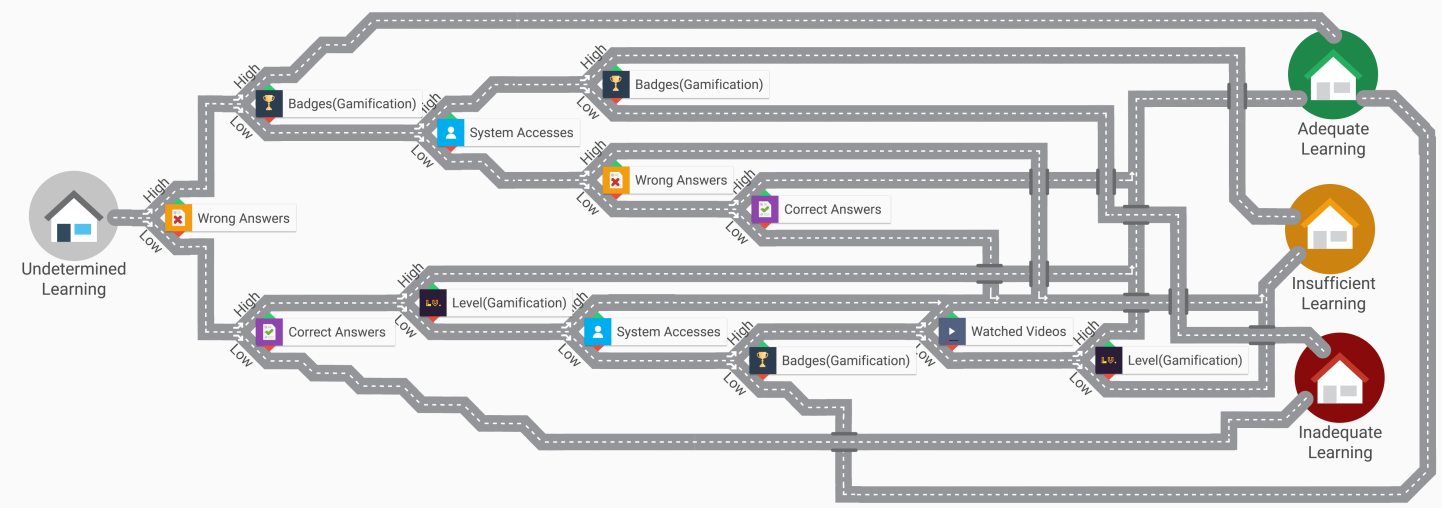

Figure 4. Viz 4 - Course of Interactions, based on WEKA's J48 output.

which alludes to the course of actions learners should take to be in the adequate class (Figure 4).

The elements of the tree image were modified: the tree was rotated $90^{\circ}$, the root node was replaced by an image that indicates the starting point (a house) and the leaf nodes, the arrival (houses colored according to the classes of result). The branches were represented by an asphalt road and intermediate nodes, by icons of educational resources with green and red arrows pointing to a direction. These arrows represent interacting more or interacting less with the resources they are associated.

\section{Design of the Experiment}

We invited professors, teachers and tutors to interact with the visualizations presented in the previous section. For each visualization, we asked them to answer some questions to check if they understood the information being displayed. After that, we asked them to express their perceptions, regarding the following metrics: (1) perceived utility $(\mathrm{PU})^{7}$ (2) perceived ease of use (PEU) ${ }^{8}$ (3) attitude towards

\footnotetext{
${ }^{7}$ Evaluates how useful the participants considered the visualizations for helping them with their professional activities

${ }^{8}$ Evaluate how easy to use the participants considered the visualizations
} 
VIII Congresso Brasileiro de Informática na Educação (CBIE 2019)

Anais do XXX Simpósio Brasileiro de Informática na Educação (SBIE 2019)

Table 1. The statements of the survey and what they measure (part 1).

\begin{tabular}{lll}
\hline$\#$ Statement & Metric \\
\hline 01 & This step would improve my work as a teacher/tutor & PU \\
02 & This step improve my effectiveness as teacher/tutor & PU \\
03 & This step would increase my productivity as a teacher/tutor & PU \\
04 & Interact at this step was clear and understandable & PEU \\
05 & I find it easy to do what I should do in this step & PEU \\
06 & Found it easy to complete this step & PEU \\
07 & This step would make work as teacher/tutor more interesting & ATU \\
08 & I would like to take this step in my daily life as a teacher/tutor & ATU \\
09 & I would use this step if it was available & IU \\
10 & This step has components with good design and style & AES \\
11 & The step design of this step is Creative & AES \\
12 & The step of this stage is aesthetically attractive & AES \\
13 & The colors (red, yellow and green) helped me understand this step & RC \\
14 & The terms (inadequate, insufficient and adequate) helped me understand & TU \\
this step & \\
\hline
\end{tabular}

Source: Based on the Technology Acceptance Model [Teo et al. 2008, Teo 2011]

use (ATU) $)^{9}$ (4) intention to use (IU) ${ }^{10}$ (5) perception about the aesthetics (AES) (6) perception about the color scheme used (RAG Colours - RC) ${ }^{12}$, (7) perception about the terms used (inadequate, insufficient, adequate) to classify students' results $(\mathrm{TU}) 13$.

These metrics were represented as statements to which the participants had to choose a value from 0 to 6 in a Likert scale, meaning: $0=$ I Strongly Disagree; 1 = I Disagree; 2 = I Slightly Disagree; $3=$ I Neither Agree nor Disagree; $4=$ I Slightly Agree; $5=$ I Agree; $6=$ I Strongly Agree (Tables ?? and 1). Participants were randomly assigned to two groups: (1) where they had to interact with multiple visualizations: the Segmented Bar Graph (Viz1), the Ordered Weights (Viz2) and the Combined Interactions (Viz3); (2) where they had to interact with only one visualization: the Course of Interactions (Viz4). After a month, we had 116 valid/complete records.

\section{Results and Discussion}

In this section, we describe the participants and the results, regarding understandability and perceptions.

\footnotetext{
${ }^{9}$ Evaluate how positive the participants are attitude regarding the use of the visualizations

${ }^{10}$ Evaluate how interested in using the visualizations the participants were, if they were available for them in their workplace

${ }^{11}$ Evaluates how beautiful and attractive the visualizations were

${ }^{12}$ Evaluates how useful/helpful were the colors used (red, yellow and green) in helping them understand the results in the visualization

${ }^{13}$ Evaluates how useful/helpful were the vocabulary used in helping them understand the results in the visualization
} 


\subsection{Participants}

In group 1, there were 53 participants (23 female and 30 male). 52 of them were between 26 and 65 years old, the majority of whom were teachers (50 participants) with a high education level (26 PhD. and 20 masters) with considerable teaching experience (15 participants had 6 to 10 years of experience and 30 participants had more than 10 years of experience), and most of them work full-time as a teacher (44 participants). Most of them use technology with their students (50 participants) and classify their computer skills as intermediate (21 participants) or advanced (31 participants). 23 participants stated they received formal training to use educational technologies. 48 participants considered themselves able to properly use educational technologies with their students.

In group 2, there were 63 participants (31 female, 31 male and 1 noninformed). 60 were between 26 and 65 years old, the majority of whom were teachers (58 participants) with a high education level (24 PhD. and 29 masters) with considerable teaching experience (14 participants had 6 to 10 years of experience and 33 participants had more than 10 years of experience), and most work full-time as a teacher (47 participants). Most participants use technology with their students (61 participants), and classify their computer skills as intermediate (23 participants) or advanced (38 participants). 28 participants stated they received formal training to use educational technologies. 60 participants considered themselves able to properly use educational technologies with their students.

\subsection{Understandability}

We asked some questions in order to check if the participants could understand the information being displayed in the visualizations. The analysis is the sum of all the correct answers, divided by the perfect score (if the participant answers all questions correctly) as the formula:

$$
\text { Understandability }=\frac{\sum_{i=1}^{n} e_{i}}{M A X_{\text {score }}}
$$

The Understandability values were high (Figure 3) for Viz1, Viz2 and Viz4 (above 90\%). This suggests these visualizations are appropriate to output educational data mining/analysis. For Viz1 ("Segmented Bar Graph"), Viz2 ("Ordered Weights") and Viz3 ("Combined Interactions"), the participants' Understandability had a median value of about 0.9 (about $90 \%$ of the questions were answered correctly), suggesting that participants were able to: (1) visualize the learners' interactions with educational resource available in the on-line environment (the objective of Viz1); (2) Compare how interactions impacted students' performance (the objective of Viz2); (3) visualize what combination of interactions promoted greater impact on students' performance (the objective of Viz3). For Viz4 ("Course of Interactions"), the participants' Understandability has a median value of $1.0(100 \%$ of the questions were answered correctly), suggesting that participants were able to visualize which sequence of interactions led students' to an inadequate, insufficient or adequate performance (the objective of Viz4). 
VIII Congresso Brasileiro de Informática na Educação (CBIE 2019)

Anais do XXX Simpósio Brasileiro de Informática na Educação (SBIE 2019)

\begin{tabular}{llll}
\hline Viz & Wilcoxon & \multicolumn{2}{l}{ Bonferroni Best } \\
\hline Viz1 vs. Viz2 & 0.0001 & $0.0007^{*}$ & Viz1 \\
\hline Viz1 vs. Viz3 & $7.217 \mathrm{e}-06$ & $4.330 \mathrm{e}-05^{*}$ & Viz1 \\
\hline Viz1 vs. Viz4 & 1.00 & - & No difference \\
\hline Viz2 vs. Viz3 & 0.012 & 0.070 & No difference \\
\hline Viz2 vs. Viz4 & $3.330 \mathrm{e}-05$ & $0.0002^{*}$ & Viz4 \\
\hline Viz3 vs. Viz4 & $1.439 \mathrm{e}-06$ & $5.759 \mathrm{e}-06^{*}$ & Viz4 \\
\hline
\end{tabular}

Table 2. Comparison between visualizations, regarding the Understandability.

Table 3. Participants' Perceptions (median/mean).

\begin{tabular}{lll}
\hline Metric & Group 1 & Group 2 \\
\hline Understandability & $0.93 / 0.84$ & $1 / 1$ \\
Attitude Towards Use & $4 / 3.94$ & $4 / 3.98$ \\
Aesthetics & $4.33 / 4.5$ & $4 / 4.09$ \\
Intention to Use & $4 / 4.14$ & $4 / 4.13$ \\
Vocabulary & $4 / 4$ & $3 / 3.78$ \\
Ease of Use & $4 / 3.7$ & $4 / 3.98$ \\
Color Scheme & $4 / 4.32$ & $4 / 3.83$ \\
Utility & $4 / 4.18$ & $3.67 / 3.81$ \\
\hline
\end{tabular}

After that, we compared the visualizations among themselves, testing for statistically significant differences regarding the Understandability. The results are: Viz4 $>$ Viz1 $>$ Viz2 $=$ Viz3 as in Table 2,

\subsection{Perceptions}

Regarding Viz1, Viz2 and Viz3, the analysis of the participants' Perceptions, the results indicate that they slightly agree (a median value between 4 and 5) that: (1) the visualizations could increase their productivity as teachers (perceived utility); (2) they are easy to use (perceived ease of use); (3) they are interesting (attitude towards use); (4) they would use these visualizations in their professional environment (intention to use); (5) they are beautiful/attractive (aesthetics); (6) the color scheme (color scheme used) is appropriate; (7) the vocabulary is appropriate (vocabulary used). See Table 3.

Regarding Viz4, the analysis of the participants' Perceptions, the results indicate that they slightly agree (a median value between 4 and 5) that: (1) the visualization is easy to use (perceived ease of use); (2) it is interesting (attitude towards use); (3) they would use them in their professional environment (intention to use); (4) they are beautiful/attractive (aesthetics); (5) the color scheme is appropriate (color scheme used). The participants neither agree nor disagree (a median value 
between 3 and 4) that the visualizations could increase their productivity as teachers (perceived utility) and that the vocabulary is appropriate (vocabulary used). See Table 3

\section{Conclusion}

We created 4 visualizations to simplify the analysis of: (1) a bar graph; (2) SimpleLogistic output; (3) JRIP output; and (4) J48 output. We evaluated the visualizations' "understandability" and the participants' perceptions, regarding: utility, ease of use, attitude towards use, intention to use, aesthetics, the color scheme used and the vocabulary used.

116 competent and experienced professors, teachers and tutors participated in the experiment. The visualization for the output of the J48 algorithm was the easiest one to understand, followed by the visualization for the bar graph, the one for the SimpleLogistic algorithm and the one for the JRIP algorithm, respectively.

The participants, overall, perceived the visualizations as slightly easy to use, interesting and beautiful/attractive and affirmed they would use it if it was available. The colors used (a RAG Colors scheme) was considered appropriate and reached its objective of grouping learners according to their results. For the perceived utility and the vocabulary used, signaling a need for improvement to clearly show the visualizations potential to help teachers making pedagogical decisions.

In general, the visualizations were effective (about $90 \%$ precision in the answers) in making teachers understand some information extracted from the outputs of educational data mining and analytics. These visualizations may provide teachers with an objective and simple to interpret glimpse of what is going on with their groups. This capacity is important to assist teachers in their daily decision-making tasks, adding value for making it evidenced by data.

We identified some topics that need further research: (1) how can we improve the visualizations' utility? (2) what kind of vocabulary is appropriate to be used? (3) are there algorithms that are easier to visualize than others? (4) what are the other algorithms we can visualize? (5) how can we visualize different information from a single educational data mining/analytics' output?

\section{References}

Alexander, M., Yuk, M., and Diamond, S. (2014). Data Visualization for Dummies. John Wiley \& Sons.

Allen, I., Seaman, J., Poulin, R., and Straut, T. (2016). Online report card: Tracking online education in the united states. Babson Park, MA: Babson Survey Research Group and Quahog Research Group, LLC.

Baker, R., Duval, E., Stamper, J., Wiley, D., and Buckingham Shum, S. (2012). Panel: educational data mining meets learning analytics. In Proceedings of 2nd International Conference on Learning Analytics $\&$ Knowledge (LAK'12), New York, USA, page 20.

Bienkowski, M., Feng, M., and Means, B. (2012). Enhancing teaching and learning through educational data mining and learning analytics: An issue brief. US Department of Education, Office of Educational Technology, pages 1-57. 
VIII Congresso Brasileiro de Informática na Educação (CBIE 2019)

Anais do XXX Simpósio Brasileiro de Informática na Educação (SBIE 2019)

Bittencourt, I. I., Costa, E., Silva, M., and Soares, E. (2009). A computational model for developing semantic web-based educational systems. Knowledge-Based Systems, 22(4):302-315.

Cohen, W. W. (1995). Fast effective rule induction. In Proceedings of the twelfth international conference on machine learning, pages 115-123.

Liyanagunawardena, T. R., Parslow, P., and Williams, S. (2014). Dropout: Mooc participants' perspective.

Onah, D. F., Sinclair, J., and Boyatt, R. (2014). Dropout rates of massive open online courses: behavioural patterns. EDULEARN14 Proceedings, pages 5825-5834.

Romero, C. and Ventura, S. (2016). Educational data science in massive open online courses. Wiley Interdisciplinary Reviews: Data Mining and Knowledge Discovery.

Schildkamp, K., Lai, M. K., and Earl, L. (2012). Data-based decision making in education: Challenges and opportunities, volume 17. Springer Science \& Business Media.

Sumner, M., Frank, E., and Hall, M. (2005). Speeding up logistic model tree induction. In European Conference on Principles of Data Mining and Knowledge Discovery, pages 675-683. Springer.

Teo, T. (2011). Factors influencing teachers' intention to use technology: Model development and test. Computers \& Education, 57(4):2432-2440.

Teo, T., Wong, S. L., and Chai, C. S. (2008). A cross-cultural examination of the intention to use technology between singaporean and malaysian pre-service teachers: An application of the technology acceptance model (tam). Educational Technology \& Society, 11(4):265-280. 